\title{
A Discussion on Current Issues for Semantic Interoperability in an Integrated Product Development Process
}

\author{
Athon Francisco Curi Staben de Moura Leite ${ }^{10000-0002-3273-5883]}$, Anderson Luis \\ Szejka ${ }^{10000-0001-8977-1351]}$, Osiris Canciglieri Junior ${ }^{10000-0002-8503-9275]}$ and Rafael Chris- \\ tiano Annunziato ${ }^{2}$ \\ ${ }^{1}$ Industrial and Systems Engineering Graduate Program, Pontifical Catholic University of Pa- \\ rana, Curitiba, Brazil \\ \{athon.leite, anderson.szejka, osiris.canciglieri\}@pucpr.br \\ ${ }^{2}$ NHS Electronic Systems, Curitiba, Brazil \\ rafaelenhs.com.br
}

\begin{abstract}
Through past decade, the complexity of integrated product development process has been significantly increased, due to highly elaborated customer's needs, market requirements and their multi-disciplinary nature. In this context, the meaning and formal structure of such information must be well managed since initial stages of product development, in order to avoid semantic heterogeneity and subsequent mistakes in interpretation. The aim of this paper is to understand the current issues related to this scenario, through the study of later works and research regarding Semantic Interoperability through ontologies in multiple domains, applicable in the field of Product Development and Manufacturing. Search material points out that a significant issue regards the input of information, since most of current models merely translate information from different sources, instead of understanding its nature and stablish a common standard to extract and structure it. Research has suggested that Knowledge Extraction models may be a viable path to filter and refine the quality of the input information, as means to improve the accuracy and effectiveness of new knowledge. This research structured its findings in a model that explores the issues of semantic interoperability through reference ontologies, to bring light to new research themes, validated through further research.
\end{abstract}

Keywords: Semantic Interoperability, Multiple Domains, Product Development and Manufacturing, Formal Models, Reference Ontology.

\section{$1 \quad$ Introduction}

In order to fulfill customer's needs, a wide range of knowledge from multiple domains is used during Product's Lifecycle Management. The complexity of client's requirements generates a remarkable quantity of information, which can be misinterpreted across different development stages and compromise the quality of final product, increasing its development and manufacturing cost. These impacts are caused 
mainly by the heterogeneity within and across different domains of knowledge in a semantic manner.

Currently, researchers are addressing the problem of semantic heterogeneity through the concept of ontology-driven semantic interoperability. This concept is based on the communication of different systems by using ontologies as means to develop a formal and shareable taxonomy that standardizes, verifies and infers information by a set of rules and axioms, improving its interpretation, traceability and integration [1]. Semantic Interoperability addresses the meaning of information exchanged in virtual environments, assuring that the same intent is captured across different systems [13]. In the context of an Integrated Product Development Process (IPDP), there are issues that challenges the quality of information flow and imposes barriers to a semantically interoperable system across multiple domains for product development and manufacturing [2] [3].

One of the most difficult challenges carried through IPDP is related to the different notations in which knowledge is represented across different development phases and domains. The necessary information is mostly structured in distinct taxonomies and extensions that are not, in most cases, designed to interoperation and further integrated applications [4].

This paper aims to analyze the current issues regarding semantic interoperability within a requirement notation perspective. Information will be gathered through the review of later works, which will be later discussed and analyzed, considering the current issues on the implementation of semantically interoperable systems in the field of integrated product development and manufacturing.

\section{Problem Statement}

The different requirements from multiple domains of knowledge might result in several a posteriori negative effects in a semantically interoperable system for product development and manufacturing. The information from each IPDP phase comes from different domains, e.g. CAD/CAM systems, CRM software, etc., having a unique notation to represent its knowledge, which requires translation [5]. This process involves restructuring information into a standard pattern for further interoperable applications, implying in issues during the requirement's analysis, such as: inconsistencies, incoherence, lack of uniqueness and unfeasible, untraceable, unverifiable information [6] [7].

In another aspect, the multiple perspectives of Product Development require a range of specialists to solve it, from different fields of study and backgrounds, using a varied set of informational tools [8]. The different experiences result in a lack of standardization during the solving process by human decisions, which may lead into misunderstanding and perpetuation of wrong assumptions for further applications [8]. In this matter, research consider increase the level of automation of the decision-making process, in order to reduce misinterpretation [9] [10] [11].

In a system's perspective, ontology-driven semantic interoperability offers a way of systems communicate by sharing knowledge from various sources [9][14]. The use of 
ontologies throughout the product design and manufacture may offer a differential in terms of standards and requirement's traceability, as long as the quality of knowledge gathering and structuring process is maintained through all development phases. By formalising this knowledge, the correct meaning of exchanged information might assure fewer mistakes and consequently reduce costs during PDP [12] [14].

Currently, semantic interoperation issues are addressed by authors, such as [12], [13] and [14], which focus on requirement's engineering (in a broader sense) in its semantically interoperable system models. The idea of such system focused on product development and manufacturing is still a theme for debate and analysis, since there are still technical and conceptual barriers that need to be overcome to a provide a seamless information interoperation [15].

\section{Related Works}

The related works of this research were structured as a qualitative literature review, based on the nature of issues in a semantically interoperable system focused on product development and manufacturing, as follows: (i) cross-domain issues, (ii) cross-IPDP phases' issues and (iii) cross-requirement representation issues.

\subsection{Cross-domain issues}

The complexity of new products demands participation of specialists from multiple domains, providing a holistic view that ensures the best solution to fulfill customers' needs [16]. Although this approach provides a better product, it ends up generating a set of heterogeneous information that represents the interests and background from different groups of stakeholders, which may not have the same meaning. This heterogeneity in information may result in divergence and misinterpretation of the correct meaning of information, due to lack of formalism and standards, affecting negatively in further product life-cycle management, by increasing costs, re-work and the overall lead time of PDP [12][17].

One of the reasons for divergence and misinterpretation resides in the expertise of specialist being restricted to their respective domains, as pointed by [18]. In addition, requirements coming from clients and software, across multiple domains, have their own structure, which results in a semantic problem that challenges the success of development process [18]. In another aspect, there is no current system capable of translate all requirements from all stakeholders in product features. This results in the necessity to manage the heterogeneous knowledge from multiple domains in different systems and product life-cycle phases, which intensifies the cross-domain issues.

Literature points to the use of different models to standardize the structure of information in different domains, such as UML (Unified Modelling Language), ModelDriven Engineering (MDE), Domain Specific Language (DSL), and others [19] [20] [21]. Although those models present an intent to formalize and represent knowledge from domains in a standard way, those approaches do not present the dynamic nature of requirements and knowledge from different product development phases [22]. 
Currently, a few models consider the consistency of requirements and performance in a dynamic environment. In [23], the authors explore a design framework for cyberphysical systems, based on design rationale, linking unique design parameters and requirements from various sources. In [24], authors explore the domain of Manufacturing and the acquisition of requirements from different domains of knowledge. Both [23] and [24] have combined different models to achieve verifiable and validated information with dynamic requirements, but still, there is the need for specialists' decisions during the translation of requirements process. That approach implies in semantic issues, as there are subjective factors and a range of different methods related to each domain, resulting in different inputs among different specialists.

\subsection{Cross-IPDP phases' issues}

The Integrated Product Development Process refers to a multi-disciplinary and communicative product development approach, which relates to the concept of Transdisciplinary Engineering applied to traditional PDP [25] [26]. There are several models and standards focused on the process, defining each of its phases, methods of verification and related tools, as pointed by [27]. However, each model is different and cannot, in an explicit way, represent all life-cycle situations in the necessary level of abstraction [28]. Although they can represent different life-cycle phases, there are particularities that make each of them more suitable for different and specific phases [27] [29].

In theory, each model is a closed loop and do follow a sequential flow of information while performing its activities. In a practical manner, though, most of the information do not follow a linear path and there is constant change in information in previous phases due to new findings in later activities [26] [27][29][30]. This end up causing inconsistencies that may increase the cost and time of development, as well as reworks and other negative impacts [30].

The communication, a basis for every integrated model, relies heavily on the semantic interpretation of each agent [26]. Across different phases, the different set of information may cause misinterpretation due to variable meanings for a term, e.g. 'orange' as a color and 'orange' as a fruit. This occurs due to the different knowledge background of developers and their experience [26][27][31]. Corroborating that affirmation, [31] states that the knowledge required for a phase of development may have different implications in later activities and can be as well changed due to the dynamic nature of product development.

Current literature, as demonstrated in [12], proposes a formalization through semantic annotations for interoperable applications, from different views in Product Life-Cycle Management. There were no annotations, however, that represented dynamic requirements. In [13], the author proposed a solution based on a model-driven ontology, which was limited to two domains and presented no evidence of further expansion and integration for more domains. 


\subsection{Cross-requirement representation issues}

Requirements are a component of every system, coming from various sources and in varied representations. In the context of product development and manufacturing, requirements may be classified as Functional (Parts Design, Functionality, etc.) and Non-Functional (International Standards, Quality objectives, Regulations, etc.) [18][32]. Those requirements represent knowledge from different stakeholders' needs, and must be assured as complete, coherent and unique, to offer a trustworthy representation of reality [32] [33].

The requirements represent the main inputs in an ontology-driven semantic interoperability system [14]. As entries to such system, all of this knowledge must be 'semantically whole', as means to avoid further interoperation issues, by means of formal structures and well defined statements [14][18]. In most cases, though, the process of abstraction of poorly defined statements end up generating divergent interpretation, which might have negative impacts related to uniqueness, comprehensiveness and, most important, traceability [14].

In [34], authors point that a correct, consistent and traceable information prevents further inconsistencies across product life-cycle. In the same research, though, authors do not provide a model to ensure requirements' traceability and no model, whatsoever on ways of optimizing the knowledge gathering and structuring process. [35] presented an interoperation model in multiple domains to ensure traceability through validation and verification methods. However, this model was limited only to early phases of a system.

Current literature, as found in [14], points out to standardized ways collected information and ensure traceable information through verification and validation. Despite that, the requirement gathering standard still relies in specialists' expertise and may require further research to develop a methodology extensible to other domains.

\section{Discussion}

One of the reasons for semantic issues occurrence in product development, as pointed out by literature, relates to the representation of requirements of a product by developers. Specialists tend to focus on a single phase of development, domain and requirement notation, e.g. the detailed design of mechanical components in a specific CAD software file. To enhance comprehension on the pointed-out issues, a model is proposed in Figure 1. Three perspectives compose the three dimensions of the problem, detailed as follows:

- Domain perspective: The Domain perspective reflects the multiple knowledge sources necessary to develop new products and its particularities;

- Integrated Product Development Process perspective: The IPDP perspective reflects the whole process of development for a new product and/or customization. Each phase may spam through different domains of knowledge and requirement notations; 
- Requirements` Notation perspective: This perspective reflects the taxonomy and modeling of the represented knowledge from different requirement sources (Design, client's specifications, among others). The Requirement Notation has significant impacts on a programming level (XML models, RDF schema, for instance) and conceptual structure (representation of product's features) across different IPDP phases and domains.

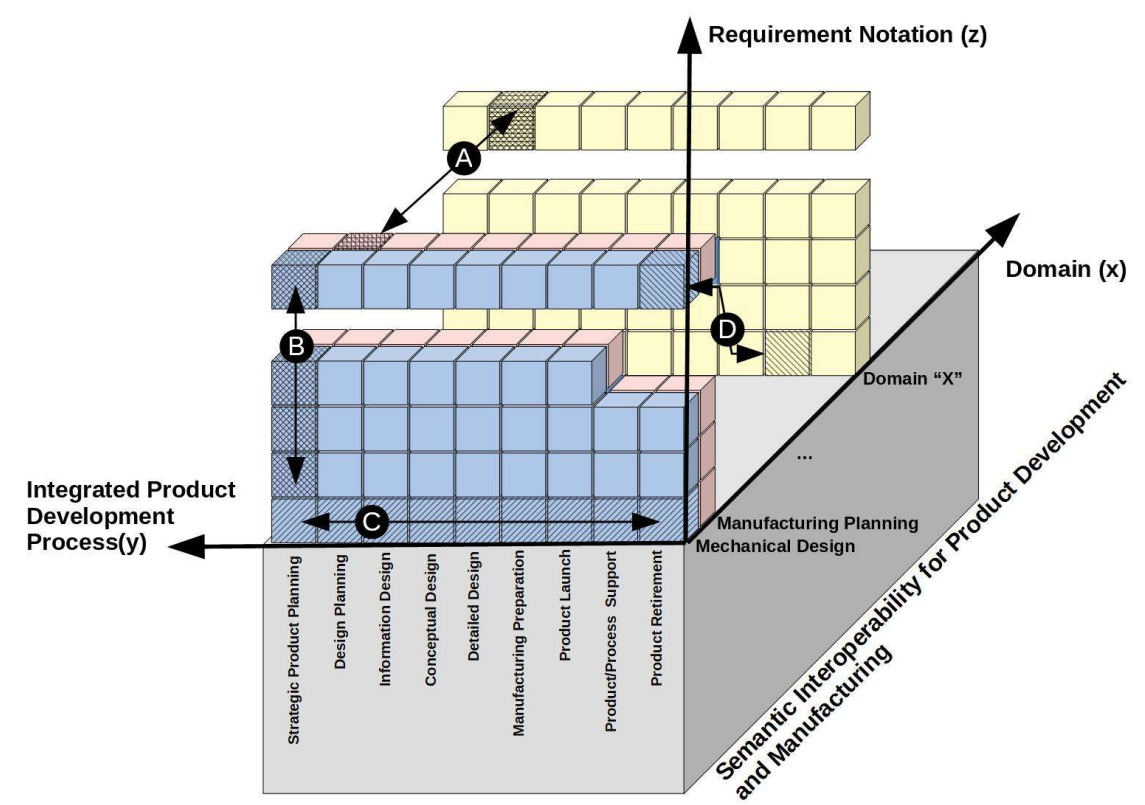

Fig. 1. Model of current issues in semantic interoperability for an integrated product development process

Each axis of the model contains one or more semantic interoperability issues related to product development and manufacturing. Each block represents a "piece" of structured knowledge. Across the 'Domain' dimension ( $\mathrm{x}$ axis), the variety of knowledge of different domains implies in heterogeneous information that may cause semantic inconsistency along the process (Detail A of Fig. 1). Within a domain and a product development phase, there is a wide range of notations ( $\mathrm{z}$ axis) to structure and represent knowledge, e.g. the product taxonomy and format of different CAD software files, presenting semantic interoperation issues on a conceptual level (Detail B of Fig. 1). Along the product development, the communication of different phases might present divergent information, resulting in issues related to information transfer, uniqueness, and traceability, among others (Detail C of Fig. 1). Last, but not least, there are the issues related to knowledge structures communicating without any similarity, which imply in lack of verifiable, trustworthy, unique, complete, coherent and traceable information (Detail D of Fig. 1). 
As depicted, each perspective (depicted in three axis) have a related issue, showing that each dimension of the model might be responsible for misinterpretations in their way. Despite the role of each of these perspectives, a complex system must consider all three operating simultaneously, to better represent a real development process. In this aspect, the fourth issue represent the relation of these perspectives, which must be considered in equal importance as the other issues.

An important observation is that this model considers as source of information the needs of consumers and developers, translated into computational language and represented as "blocks" of structured knowledge. The dynamic nature of such requirements characterizes the complexity of managing and sharing such information without losing the original meaning.

The proposed model is a representation of the current issues on Semantic Interoperability for Product Development and Manufacturing, by a Requirement Notation perspective. Such model can provide a holistic view to the problems presented on selected research and act as a starting point for further research on the area. Further applications on semantic interoperability might be benefited from the model, as it provides a clear view on the current issues that might be addressed through new computational systems and methodologies.

As an inference on the model, the current issues for semantic interoperability in integrated product development process occur, in general, due to systems that heavily rely on the expertise of specialists and developers. As different specialists create singular solutions, it is necessary to develop a standardized way of collecting and structuring knowledge from products' requirements. Thus, such formal approach should benefit from a deeper study on the information flows and relations between domains.

Issues related to the requirements' notation, as pointed out by literature, are still not well defined and do not present a formal and effective method for solution. The input of information has significant impacts on the final product and should be cautiously gathered. In this sense, a knowledge extraction model for semantic interoperability would benefit the final quality and completeness of information.

\section{Conclusion}

This paper presented a research that led to a model for current issues on semantic interoperability within an integrated product development process context. The model offers a fresh view on several issues related to semantically interoperable system through a requirement notation perspective, offering new questions for further research.

Current literature points out several challenging issues that need to be addressed to provide seamless information interoperation. Authors are already developing solutions, but there are still points to be observed in further research. In future applications, the notation aspect of requirements may be a differential aspect which will improve products, customers` satisfaction and reduce costs.

The high level of abstraction and needs for specialists may be a challenge to overcome in further models. By an input perspective for product life-cycle, a model to 
improve and increase automation in decision-making processes related to knowledge gathering and structuring is still necessary.

An aspect of this research, noted throughout its development is that further literature review by a systematic approach is necessary, to validate and define the state-of-the-art on related topics of study, covering all recent and relevant literature.

In order to expand this research further works are suggested, such as a systematic literature review on semantic interoperability through requirements' notation perspective in PLM, development of a knowledge extraction model for semantic interoperability in IPDP and an integration of knowledge extraction models and a semantic reconciliation process.

\section{References}

1. Chungoora, N., Canciglieri, O. \& Young, R. I. M. Towards expressive ontologybased approaches to manufacturing knowledge representation and sharing. Int. J. Comput. Integr. Manuf. 23, 1059-1070 (2010).

2. Canciglieri Jr. O., Young R.I.M.: Information sharing in multiviewpoint injection moulding design and manufacturing. International Journal of Production Research. 41, (7), 1565-1586 (2003).

3. Anjum, N. et al. Verification of knowledge shared across design and manufacture using a foundation ontology. Int. J. Prod. Res. 51, 6534-6552 (2013).

4. Chen, D., Doumeingts, G. \& Vernadat, F. Architectures for enterprise integration and interoperability: Past, present and future. Comput. Ind. 59, 647-659 (2008).

5. Chungoora, N. \& Young, R. I. M. Semantic reconciliation across design and manufacturing knowledge models: A logic-based approach. Appl. Ontol. 6, 295315 (2011).

6. Szejka, A. L., Canciglieri Jr., O., Loures, E. R., Panetto, H., Aubry, A. Requirements interoperability method to support integrated product development, in: 45th Computers and Industrial Engineering, 147, 1-8 (2015).

7. Imran, M. \& Young, R. I. M. The application of common logic based formal ontologies to assembly knowledge sharing. J. Intell. Manuf. 26, 139-158 (2013).

8. Song, W., Ming, X. \& Wang, P. Collaborative product innovation network: Status review, framework, and technology solutions. Concurr. Eng. 21, 55-64 (2013).

9. Schmidt, D.C.: Model-Driven Engineering. IEEE Computer. 39, (2), 25-31 (2006).

10. Strasunskas, D., Hakkarainen, S.E.: Domain model-driven software engineering: A method for discovery of dependency links. Information and Software Technology. 54, (11), 1239- 1249 (2012).

11. Barbieri, G., Fantuzzi, C., Borsari, R.: A model-based design methodology for the development of mechatronic systems. Mechatronics - In Press, (2014).

12. Liao, Y., Lezoche, M., Panetto, H., Boudjlida, N. \& Loures, E. R. Semantic annotation for knowledge explicitation in a product lifecycle management context: A survey. Comput. Ind. 71, 24-34 (2015).

13. Chungoora, N., Young, R.I., Gunendran, G., Palmer C., Usman, Z., Anjum, N.A., CuttingDecelle A.F., Harding, J.A., Case, K.: A model-driven ontology approach for manufacturing system interoperability and knowledge sharing. Computers in Industry. 64, (4), 392-401 (2013). 
14. Szejka, A.L., Canciglieri Jr., O., Loures, E.R., Panetto, H., Aubry, A. Proposal of a model-driven ontology for product development process interoperability and information sharing, Lecture Notes Comput. Sci., , (2016).

15. Chungoora, N. \& Young, R. I. M. The configuration of design and manufacture knowledge models from a heavyweight ontological foundation. Int. J. Prod. Res. 49, 4701-4725 (2011).

16. Pereira, J.A., Canciglieri Jr., O., Lazzaretti, A.E., Souza, P.M. Application of inte grated product development model oriented to R\&D projects of the Brazilian electricity sector, Adv. Mater. Res., 945-949, 401-409 (2014).

17. Stechert, C., Franke, H.J.: Managing requirements as the core of multi-disciplinary product development. CIRP Journal of Manufacturing Science and Technology. 1, (3), 153-158 (2009).

18. Szejka, A. L., Aubry, A., Panetto, H., Canciglieri Jr., O. Loures, E. R. Towards a conceptual framework for requirements interoperability in complex systems engineering. Lecture Notes on Computer Science, LNCS 8842, 229-240 (2014).

19. Canciglieri Jr. O., Young R.I.M.: Information sharing in multiviewpoint injection moulding design and manufacturing. International Journal of Production Research. 41, (7), 1565-1586 (2003).

20. Schmidt, D.C.: Model-Driven Engineering. IEEE Computer. 39, (2), 25-31 (2006).

21. Haveman, S.P., Bonnema G.M.: Requirements for High Level Models Supporting Design Space Exploration in Model-based Systems Engineering. Procedia Computer Science. 16, 293-302 (2013).

22. Nattermann, R., Reiner, A.: Approach for a data-management-system and a proceedingmodel for the development of adaptronic systems. In: International mechanical engineering congress and exposition (ASME), pp. 1-10, Vancouver (2010).

23. Moneva, H., Hamberg, R. Punter, T.: A Design Framework for Model-Based Development of Complex Systems. In: 32nd IEEE Real-Time Systems Symposium and 2nd Analytical Virtual Integration of Cyber-Physical Systems Workshop, pp. 1-8, Vienna, (2011).

24. Chungoora, N. et al. A model-driven ontology approach for manufacturing system interoperability and knowledge sharing. Comput. Ind. 64, 392-401 (2013).

25. Rozenfeld, H., Forcellini, F.A., Amaral, D.C., Toledo, J.C., Silva, S.L., Alliprandini, D.H., Scalice, R.K. Gestão de Desenvolvimento de Produtos: Uma Referência Para a Melhoria do Processo, Saraiva, São Paulo (2006).

26. Stechert, C., Franke, H.J.: Managing requirements as the core of multi-disciplinary product development. CIRP Journal of Manufacturing Science and Technology. 1, (3), 153-158 (2009).

27. Pereira, J.A., Canciglieri Jr., O. Product development model oriented for the R\&D projects of the Brazilian electricity sector, Appl. Mech. Mater. 518, 366-373 (2014).

28. de Moura Leite, AFCSM, Canciglieri, M. B., Szejka, A. L., Canciglieri Jr., O. The reference view for semantic interoperability in Integrated Product Development Process: The conceptual structure for injecting thin walled plastic products. Journal of Industrial Information Integration, 7, 13-23 (2017). 
29. Young, R.I.M., Gunendran, A.G., Cutting-Decelle, A.F., Gruninger, M. Manufacturing knowledge sharing in PLM: a progression towards the use of heavy weight ontologies, Int. J. Product. Res. 45, (7), 1505-1519 (2007).

30. Kim, K.-Y., Manley, D. G. \& Yang, H. Ontology-based assembly design and information sharing for collaborative product development. Comput. Des. 38, 1233-1250 (2006).

31. Nattermann, R., Reiner, A.: Approach for a data-management-system and a proceedingmodel for the development of adaptronic systems. In: International mechanical engineering congress and exposition (ASME), pp. 1-10, Vancouver (2010).

32. Ramesh, B., Jarke, M.: Toward reference models for requirements traceability. IEEE Transactions on Software Engineering. 27, 58-93 (2001).

33. Spanoudakis, G., Zisman, A., Pérez-Miñana, E., Krause P.: Rule-based generation of requirements traceability relations. Journal of Systems and Software. 72, (2), 105-127 (2004).

34. Pohl, K.: The three Dimensions of Requirements Engineering: A framework and its applications. Informatic Systems. 19, (3), 243-258 (1994).

35. Cleland-Huang, J., Chang, C.K., Sethi, G., Javvaji, K., Haijian, H., Jinchun, X.: Automating speculative queries through event-based requirements traceability. In: Proceedings of IEEE Joint International Conference on Requirements Engineering, pp.289-296 (2002). 